J. Austral. Math. Soc. 19 (Series A), (1975), 74-90.

\title{
CONTRACTORS AND SECANT METHODS FOR FINDING ROOTS OF NON-LINEAR FUNCTIONALS
}

\author{
MIECZYSLAW ALTMAN \\ (Received 28 August 1973) \\ Communicated by B. Mond
}

\begin{abstract}
A further development of the concept of contractors for non-linear functionals is presented by introducing two-point contractors. On this basis two-point secant metlods are proposed for finding roots of non-linear functionals in Banach spaces.
\end{abstract}

\section{Introduction}

In this paper we apply the idea of two-point secant methods to the problem of finding approximate roots of non-linear functionals. For this purpose a further development of the contractor concept for non-linear functionals in Banach spaces is presented. An analysis of the contractor notion applied to two-point secant methods for non-linear operators makes it clear how to generalise the contractor concept for non-linear functionals. In this way the notion of a two-point contractor for a non-linear functional is introduced. Let us recall that the contractor notion for a non-linear functional has been introduced independently in a sense that the definition of a contractor for a non-linear functional is not a simple specialization of the same definition of a contractor for a non-linear operator. In the same way the definition of a two-point contractor for a non-linear functionel is being introduced independently. On the basis of the concept of two-point contractors for non-linear functionals various two-point iteration methods can be introduced for finding roots of non-linear functionals. By analogy these methods are called twopoint secant methods for non-linear functionals.

Given a Banach space $X$ and a non-linear functional $F: D \subset X \rightarrow R$ (reals) let $Q(s, t) \geqq 0$ for $t, s \geqq 0$. For simplicity we can assume that $D$ is a closed sphere $S=S\left(x_{0}, r\right)$ with centre $x_{0}$ and radius $r$.

Definition 1.1. A mapping $\Gamma: D \times D \rightarrow X$ is said to be a two-point contractor for $F$ with majorant function $Q$ if the following inequality is satisfied 


$$
|F(x+t \Gamma(x, \bar{x}))-F x-t| \leqq Q(|t|,\|x-\bar{x}\|)
$$

for $x, \bar{x} \in D$ and real $t$ whenever $x+t \Gamma(x, \bar{x}) \in D-$ the domain of $F$. Inequality (1.1) is called the contractor inequality.

Let us recall that a contractor for $F$ as defined in Altman (to appear) is a mapping $\Gamma: D \rightarrow X$. Thus, the concept of a two-point contractor for a non-linear functional $F$ generalises the concept of a contractor for $F$. The following contractor inequality is a special case of (1.1)

$$
|F(x+t \Gamma(x, \bar{x}))-F x-t| \leqq o(|t|)+K\|x-\bar{x}\|,
$$

where $K$ is a constant and $0 \leqq o(s) / s \rightarrow 0$ as $s \rightarrow 0, s \geqq 0$. It follows from $\left(1.1^{*}\right)$ that $\tilde{\Gamma}(x)=\Gamma(x, x)$ is an inverse derivative for $F$ as defined in Altman (1973) and, consequently, a contractor in the sense of Altman (1973).

By means of analogy the first divided difference functional of $F$ is a linear bounded functional

$$
G: D \times D \rightarrow X^{*} \text { (the conjugate space) }
$$

which satisfies the relationship

$$
G(x, \bar{x})(x-\bar{x})=F x-F \bar{x} \text { for } x, \bar{x} \in D \subset X .
$$

For each $x, \bar{x} \in D$ choose $y \in X$ such that $G(x, \bar{x}) y \neq 0$ and put $\Gamma(x, \bar{x})=y / G(x, \bar{x}) y$.

LEMMA 1.1. If $\|\Gamma(x, \vec{x})\| \leqq B$ with some constant $B$ and

$$
\|G(x, \bar{x})-G(\xi, \bar{\xi})\| \leqq K[\|x-\xi\|+\|x-\bar{x}\|]
$$

for $x, \bar{x}, \xi, \bar{\xi} \in D$, then $\Gamma(x, \bar{x})$ is a two-point contractor for $F$.

Proof. Put $h=t \Gamma(x, \bar{x}) ;$ then we obtain

$$
\begin{aligned}
& |F(x+h)-F x-t|=|F(x+h)-F x-G(x, \bar{x}) h| \\
& \quad=|G(x+h, x) h-G(x, \bar{x}) h| \leqq K[\|h\|+\|x-\bar{x}\|]\|h\| \\
& \leqq K B(B|t|+\|x-\bar{x}\|)|t|=Q(|t|,\|x-\bar{x}\|) .
\end{aligned}
$$

Note that Lemma 1.1 remains true if one replaces condition (1.2) by the following one

$$
|F x-F \bar{x}-G(\xi, \bar{x})(x-\bar{x})| \leqq K\|x-\bar{x}\| \cdot\|x-\xi\| .
$$

In fact, we have for $h=t \Gamma(x, \bar{x})$

$$
\begin{aligned}
& |F(x+h)-F x-t|=|F(x+h)-F x-G(x, \bar{x}) h| \\
& \quad \leqq K\|h\| \cdot\|x+h-\bar{x}\| \leqq K\|h\|(\|h\|+\|x-\bar{x}\|) \\
& \leqq K B|t|(B|t|+\|x-\bar{x}\|)=Q(|t|,\|x-\bar{x}\|) .
\end{aligned}
$$


A two-point contractor is said to be bounded if there exists a constant $B$ such that

$$
\|\Gamma(x, \bar{x})\| \leqq B \text { for } x, \bar{x} \in D \subset X .
$$

Consider the equation

$$
F x=0,
$$

where $F: D \rightarrow R$ is a closed non-linear functional, i.e., $x_{n} \in D, x_{n} \rightarrow x$ and $F x_{n} \rightarrow c$ imply $x \in D$ and $F x=c$.

We assume that $F$ has a two-point contractor $\Gamma$ satisfying the contractor inequality (1.1) with $Q(s, t)$ continuous and non-decraasing in each variable and $Q(0,0)=0$.

In order to solve (1.4) we consider the following general iterative procedure

$$
x_{n+1}=x_{n}-F x_{n} \Gamma\left(x_{n}, \bar{x}_{n}\right), \quad n=0,1, \cdots,
$$

where $\bar{x}_{n}$ will be defined below. We consider also the iterative numerical sequence $\left\{t_{n}\right\}$ defined as follows

$$
t_{n+1}=t_{n}+Q\left(t_{n}-t_{n-1}, C\left(t_{n}-t_{n-1}\right)\right), \quad n=0,1, \cdots
$$

with initial values $t_{0}=0, t_{1}=Q(\eta, C \eta)$, the constant $C$ being defined below. We investigate the iterative procedure (1.5) under the assumption that the elements $\bar{x}_{n}$ are chosen so as to satisfy

$$
\left\|x_{n}-\bar{x}_{n}\right\| \leqq C\left|F x_{n}\right|, \quad n=0,1, \cdots
$$

Assume also that the sequence $\left\{t_{n}\right\}$ determined by (1.6) has a limit $t^{*}$

THEOREM 1.1. Let $F: D \rightarrow R$ be a closed non-linear functional with domain $D$ containing the sphere $S=S\left(x_{0}, r\right)$, where $x_{0}$ is such that $\left|F x_{0}\right| \leqq \eta$. Suppose that $\Gamma: S \times S \rightarrow X$ is a two-point contractor for $F$ satisfying (1.3) and the contractor inequality (1.1) for $x, \bar{x} \in S$ and real $t$ whenever $x+t \Gamma(x, \bar{x}) \in D$. Let $\bar{x}_{n}$ in (1.5) be chosen so as to satisfy (1.7). Assume also that the sequence $\left\{t_{n}\right\}$ defined by (1.5) has a limit $t^{*}$ and $r=B t^{*}$. Then the sequence $\left\{x_{n}\right\}$ defined by (1.5) and (1.7) converges to a solution $x$ of equation (1.4). All $x_{n}$ lie in $S$ and the following error estimate holds

$$
\left\|x-x_{n}\right\| \leqq B\left(t^{*}-t_{n}\right), \quad n=0,1, \cdots
$$

Proof. It follows from the contractor inequality (1.1) with $t=-F x_{n}$, $x=x_{n}, \bar{x}=\bar{x}_{n}$ and in virtue of (1.5) using also (1.7) that

$$
\left|F x_{n+1}\right| \leqq Q\left(\left|F x_{n}\right|, C\left|F x_{n}\right|\right), \quad n=0,1, \cdots
$$

Now we show by induction that 


$$
\left|F x_{n}\right| \leqq t_{n}-t_{n-1} \text { for } n=1,2, \cdots
$$

In fact, using (1.9) with $n=0$ we obtain

$$
\left|F x_{1}\right| \leqq Q\left(\left|F x_{0}\right|, C\left|F x_{0}\right|\right) \leqq Q(\eta, C \eta)=t_{1}-t_{0},
$$

in virtue of (1.6). Assuming $\left(1.10_{n}\right)$ and using (1.9) again we conclude in virtue of (1.6) that

$$
\left|F x_{n+1}\right| \leqq Q\left(t_{n}-t_{n-1}, C\left(t_{n}-t_{n-1}\right)\right)=t_{n+1}-t_{n}
$$

whence $\left(1.10_{n+1}\right)$ follows.

Furthermore, (1.3), (1.5) and $\left(1.10_{n}\right)$ imply

that is

$$
\begin{aligned}
& \left\|x_{n+m}-x_{n}\right\| \leqq \sum_{i=n}^{n+m-1}\left\|x_{i+1}-x_{i}\right\| \leqq B \sum_{i=n}^{n+m-1}\left|F x_{i}\right| \\
& \leqq B \sum_{i=n}^{n+m-1}\left(t_{i+1}-t_{i}\right)=B\left(t_{n+m}-t_{n}\right),
\end{aligned}
$$

$$
\left\|x_{n+m}-x_{n}\right\| \leqq B\left(t_{n+m}-t_{n}\right) .
$$

Inequality (1.11) shows that $\left\{x_{n}\right\}$ is a Cauchy sequence and, consequently, has a limit $x$. Since $F x_{n} \rightarrow 0$ as $n \rightarrow \infty$, by $\left(1.10_{n}\right)$, and $F$ is closed, we conclude that $F x=0$. Putting $n=0$ in (1.11) we obtain

$$
\left\|x_{m}-x_{0}\right\| \leqq B t^{*} \text { for } m=1,2, \cdots
$$

Letting $m \rightarrow \infty$ in (1.11) we obtain the error estimate (1.8) and the proof is complete.

Now let us discuss some special cases of condition (1.7) in conjunction with procedure (1.5).

Suppose that $\bar{x}_{n}$ in (1.5) is defined as follows

$$
\bar{x}_{n+1}=x_{n+1}-F x_{n+1} \Gamma\left(x_{n}, \bar{x}_{n}\right), \quad n=0,1, \cdots
$$

Assuming (1.3) we conclude from (1.12) that condition (1.7) is satisfied with constant $C=B$. Suppose now that the majorant function $Q$ has the form $Q(s, t)$ $=K B(B s+t) s$, where $B, K$ are some positive constants. Thus, the contractor inequality (1.1) becomes

$$
|F(x+t \Gamma(x, \bar{x}))-F x-t| \leqq B K(B|t|+\|x-\bar{x}\|)|t| .
$$

In this case the procedure defined by (1.5) and (1.12) is quadratically convergent and we have the following.

THEOREM 1.2. Let $F: D \rightarrow R$ be a closed non-linear functional with domain $D$ containing the sphere $S=S\left(x_{0}, r\right)$, where $\left|F x_{0}\right| \leqq \eta,\left\|x_{0}-\bar{x}_{0}\right\| \leqq B \eta$, $a=2 K B^{2}, q=a \eta<1, r=B \eta t^{*}$ and $t^{*}=\sum_{n=0}^{\infty} q^{2^{n-1}}$. 
Suppose that $\Gamma$ is a two-point contractor for $F$ satisfying (1.3) and the contractor inequality (1.13) for $x, \bar{x} \in S$ whenever $x+t \Gamma(x, \bar{x}) \in S$.

Then the sequence $\left\{x_{n}\right\}$ defined by (1.5) and (1.12) converges to a solution $x$ of the equation $F x=0$. All $x_{n}$ lie in $S$ and the error estimate holds:

$$
\left\|x-x_{n}\right\| \leqq B \eta t^{*} q^{2^{n-1}} \text {. }
$$

Proof. It follows from the contractor inequality (1.13) with $x=x_{n}, \bar{x}=\bar{x}_{n}$ and $t=-F x_{n}$ that

$$
\left|F x_{n+1}\right| \leqq 2 K B^{2}\left|F x_{n}\right|^{2} \leqq a t_{n}^{2}=t_{n+1}
$$

where $t_{0}=\eta, n=1,2, \cdots$ and $\left|F x_{1}\right| \leqq a \eta^{2}=t_{1}$. We prove by induction that

$$
t_{n}=\eta q^{2^{n-1}} \text { where } t_{n+1}=a t_{n}^{2}, \quad n=0,1, \cdots
$$

Hence, in virtue of (1.5), (1.3), (1.15) and (1.16) we obtain

$$
\begin{aligned}
& \left\|x_{n+1}-x_{n}\right\| \leqq B \eta q^{2^{n-1}} \\
& \left\|x_{n+1}-x_{0}\right\| \leqq B \eta \sum_{i=0}^{n} q^{2^{i-1}}<B \eta t^{*} .
\end{aligned}
$$

The latter inequality shows that all $x_{n}$ lie in $S$. Further, we have

$$
\left\|x_{n+m}-x_{n}\right\| \leqq B \eta \sum_{i=n}^{n+m-1} q^{2^{i}-1}<B \eta t^{*} q^{2^{n-1}} .
$$

Thus, $\left\{x_{n}\right\}$ is a Cauchy sequence and has a limit $x$. It follows from (1.15), (1.16) and $q<1$ that $F x_{n} \rightarrow 0$ as $n \rightarrow \infty$. Since $F$ is closed, we conclude that $F x=0$. The error estimate (1.14) follows from (1.17) by letting $m \rightarrow \infty$.

We assume now that the first divided difference functional $G$ for $F$ exists and satisfies condition (1.2) or $\left(1.2^{*}\right)$ on $S=S\left(x_{0}, r\right) \subset D$. Under these hypotheses Lemma 1.1 shows that $\Gamma(x, \bar{x})=y / G(x, \bar{x}) y$, where $y \in X$ is such that $G(x, \bar{x}) y \neq 0$, is a bounded two-point contractor for $F$ provided that

$$
\|\Gamma(x, \bar{x})\| \leqq B \text { for } x, \bar{x} \in S
$$

holds true. Let us consider the following iterative procedure

$$
\begin{aligned}
& x_{n+1}=x_{n}-F x_{n} \Gamma\left(x_{n}, \bar{x}_{n}\right) \\
& \bar{x}_{n+1}=x_{n+1}-F x_{n+1} \Gamma\left(x_{n}, \bar{x}_{n}\right)
\end{aligned}
$$

for $n=0,1, \cdots$

Applying Theorem 1.2 to this procedure we obtain

THEOREM 1.3. Let $F: D \rightarrow R$ be a closed non-linear functional with domain $D$ containing the sphere $S$. Suppose that there exists the first divided difference functional $G$ for $F$ satisfying (1.2) or (1.2*) and (1.18). Let the initial elements $x_{0}$ 
and $\bar{x}_{0}$ be chosen so as to satisfy $\left|F x_{0}\right| \leqq \eta,\left\|x_{0}-\bar{x}_{0}\right\| \leqq B \eta, r=B \eta t^{*}, a=2 K B^{2}$, $q=a \eta<1$ and $t^{*}=\sum_{n=0}^{\infty} q^{2^{n-1}}$. Then the sequence $\left\{x_{n}\right\}$ defined by (1.19) and (1.20) converges to a solution $x$ of the equation $F x=0$. All $x_{n}$ lie in $S$ and the error estimate (1.14) holds.

Proof. In virtue of Lemma $1.1 \Gamma$ is a two-point bounded contractor for $F$ satisfying the contractor inequality (1.13). It is easy to see that all hypotheses of Theorem 1.2 are satisfied. Hence, the proof follows immediately.

REMARK 1.1. It is sufficient to assume instead of (1.18) that

$$
\left\|\Gamma\left(x_{n}, \bar{x}_{n}\right)\right\|=\left\|y_{n}\right\| /\left|G\left(x_{n}, \bar{x}_{n}\right) y_{n}\right| \leqq B \text { for } n=0,1, \cdots
$$

\section{Two-point contractors and consistent approximations}

Consistent approximations were introduced by Ortega (see Ortega and Rheinbolt (1970), NR 11.2-3. page 363) as a more general notion than a divided difference operator of a non-linear operator. The case discussed here is related to non-linear functionals. It turns out that the notion of consistent approximations is a particular case of the two-point contractor concept for non-linear functionals.

Let $F: D \rightarrow R$ be a non-linear functional with Fréchet derivative $F(x)$, $x \in D \subset X$.

Definition 2.1. A mapping $J: D \times D \rightarrow X^{*}$ is called a strongly consistent approximation to $F^{\prime}$ on $S=S\left(x_{0}, r\right) \subset D$ if there exists a constant $c$ such that

$$
\left\|F^{\prime}(x)-J(x, \bar{x})\right\| \leqq c\|x-\bar{x}\| \text { for } x, \bar{x} \varepsilon S .
$$

Lemma 2.1. Let $F: D \rightarrow R$ be continuously differentiable in $S$ and let the Fréchet derivative $F^{\prime}$ be Lipschitz continuous on $S$ with Lipschitz constant $R$. Suppose that $J$ is a strongly consistent approximation to $F^{\prime}$ satisfying (2.1). For each $x, \bar{x} \varepsilon S$ choose $y \varepsilon X$ such that $J(x, \bar{x}) y \neq 0$. Then

$$
J(x, \bar{x}) y, \quad x, \bar{x} \varepsilon S
$$

is a two-point bounded contractor for $F$ provided that $\Gamma$ satisfies the condition

$$
\|\Gamma(x, \bar{x})\| \leqq B \text { for } x, \bar{x} \varepsilon S,
$$

where $B$ is some constant.

ProOF. For $h=t \Gamma(x, \bar{x})$ we have

$$
\begin{aligned}
& |F(x+t \Gamma(x, \bar{x}))-F x-t| \leqq\left|F(x+h)-F x-F^{\prime}(x) h\right| \\
& \quad+\left|F^{\prime}(x) h-J(x, \bar{x}) h\right| \leqq \frac{1}{2} R\|h\|^{2}+c\|x-\bar{x}\| \cdot\|h\| \\
& \quad \leqq \frac{1}{2} K B^{2} t^{2}+c B\|x-\bar{x}\| \cdot|t|
\end{aligned}
$$


in virtue of (2.1) and (2.3). Hence, we obtain the following contractor inequality

$$
|F(x+t \Gamma(x, \bar{x}))-F x-t| \leqq B K(B|t|+\|x-\bar{x}\|)|t|
$$

for $x, \bar{x} \varepsilon S$ whenever $x+t \Gamma(x, \bar{x}) \varepsilon S$, where $K=\max \left(\frac{1}{2} K, c\right)$.

THEOREM 2.1. Let $F: D \rightarrow R$ be a non-linear continuously differentiable functional with domain $D$ containing $S$ and let $F^{\prime}$ be Lipschitz continuous on $S$ with Lipschitz constant $\bar{K}$. Let $J$ be a strongly consistent approximation to $F^{\prime}$ satisfying (2.1) and (2.3). Suppose that $x_{0}, \bar{x}_{0}$ are chosen so as to satisfy $\left|F x_{0}\right| \leqq \eta$, $\left\|x_{0}-\bar{x}_{0}\right\| \leqq B \eta, a=2 K B^{2}, q=a \eta<1, r=B \eta t^{*}$ and $t^{*}=\sum_{n=0}^{\infty} q^{2^{n-1}}$, where $K=\max \left(\frac{1}{2} \bar{K}, c\right)$. Then the sequence $\left\{x_{n}\right\}$ defined by (1.5) and (1.12) with $\tilde{\Gamma}$ determined by (2.2) converges to a solution $x$ of $F x=0$. All $x_{n}$ lie in $S$ and the error estimate (1.14) holds true.

Proof. Since the hypotheses of Lemma 2.1 are satisfied, it follows that $\Gamma$ defined by (2.2) is a two-point bounded contractor for $F$ satisfying the contractor inequality (2.4). It is easily seen that all hypotheses of Theorem 1.2 are satisfied and the proof follows immediately.

REMARK 2.1. Let us observe that condition (2.3) can be replaced by the following one

$$
\left\|\Gamma\left(x_{n}, \vec{x}_{n}\right)\right\|=\left\|y_{n}\right\| /\left|J\left(x_{n}, \bar{x}_{n}\right) y_{n}\right| \leqq B \text { for } n=0,1, \cdots
$$

Remark 2.2. Let $0<\beta<1$ be any fixed number. One can choose $y$ in (2.2) so as to satisfy the following conditions

$$
J(x, \bar{x}) y \geqq \beta\|J(x, \bar{x})\|,\|y\|=1 .
$$

Now assume that there exists a constant $\bar{B}$ such that

$$
1 /\|J(x, \bar{x})\| \leqq \bar{B} \text { for } x, \bar{x} \& S .
$$

Then we obtain in virtue of (2.5), (2.6)

$$
\|\Gamma(x, \bar{x})\| \leqq \bar{B} / \beta \text { for } x, \bar{x} \varepsilon S .
$$

Thus, in all cases condition (2.3) can be replaced by (2.7).

In some Banach spaces one can put $\beta=1$. This is the case, for instance, in reflexive Banach spaces.

\section{Two-point contractors bounded by functions}

In this paragraph we discuss iterative procedures involving two-point contractors bounded by functions. We also introduce a more general majorant function.

Let $F: D \rightarrow R$ be a non-linear closed functional with domain $D$ containing the sphere $S=S\left(x_{0}, r\right)$ with radius $r$ to be defined below. Furthermore, let 
$Q(s, \bar{s}, t, \bar{t}) \geqq 0$ for $s, \bar{s}, t, \bar{t} \geqq 0$ be a continuous function which is non-decreasing in each variable.

Let $\Gamma: D \times D \rightarrow X^{*}$ be a two-point contractor for $F$ satisfying the following contractor inequality

$$
\begin{aligned}
& |F(x+t \Gamma(x, \bar{x}))-F x-t| \\
& \leqq Q\left(\|t \Gamma(x, \bar{x})\|,\|x+t \Gamma(x, \bar{x})-\bar{x}\|,\left\|x-x_{0}\right\|,\left\|\bar{x}-\bar{x}_{0}\right\|\right)
\end{aligned}
$$

for $x, \bar{x} \varepsilon S$ whenever $x+t \Gamma(x, \bar{x}) \varepsilon S$.

Let $B(t, \bar{t}) \geqq 0$ for $t, \bar{t} \geqq 0$ be a function which is non-decreasing in each variable. We assume that the following estimate holds true for the contractor $\Gamma$

$$
\|\Gamma(x, \bar{x})\| \leqq B\left(\left\|x-x_{0}\right\|,\left\|\bar{x}-\bar{x}_{0}\right\|\right)
$$

for $x, \bar{x} \varepsilon S$ with $x_{0} \varepsilon S$ being fixed.

Now consider the general two-point contractor procedure

$$
x_{n+1}=x_{n}-F x_{n} \Gamma\left(x_{n}, \bar{x}_{n}\right), \quad n=0,1, \cdots,
$$

where $\bar{x}_{n}$ are chosen so as to satisfy the following inequalities

$$
\begin{aligned}
& \left\|x_{n+1}-\bar{x}\right\| \leqq \gamma_{1}\left\|x_{n+1}-x_{n}\right\| \\
& \left\|\bar{x}_{n}-\bar{x}_{0}\right\| \leqq \gamma_{2}\left\|x_{n}-x_{0}\right\|
\end{aligned}
$$

for $n=0,1, \cdots, \gamma_{1}$ and $\gamma_{2}$ being constant numbers. We also consider the numerical sequence $\left\{t_{n}\right\}$ defined by the following iterative process

$$
t_{n+1}=t_{n}+B\left(t_{n}, \gamma_{2} t_{n}\right) Q\left(t_{n}-t_{n-1}, \gamma_{1}\left(t_{n}-t_{n-1}\right), t_{n-1}, \gamma_{2} t_{n-1}\right),
$$

$n=0,1, \cdots$ with initial value $t_{0}=0$.

We assume that

$$
t_{n} \rightarrow t^{*} \text { as } n \rightarrow \infty \text {. }
$$

THEOREM 3.1. Suppose that $F: D \rightarrow R$ is a closed non-linear functional with domain D containing the sphere $S$ and $x_{0}$ is such that

$$
\left\|F x_{0} \Gamma\left(x_{0}, \bar{x}_{0}\right)\right\| \leqq \eta=t_{1} \text { and } r=t^{*} .
$$

If $\Gamma$ is a two-point contractor for $F$ satisfying (3.1) and (3.2) where $Q$ is continuous and non-decreasing in each variable with $Q\left(0,0, t^{*}, \gamma_{2} t^{*}\right)=0$, then all $x_{n}$ lie in $S$ and the sequence $\left\{x_{n}\right\}$ defined by (3.3)-(3.5) converges to a solution $x$ of equation $F x=0$ and the error estimate holds

$$
\left\|x-x_{n}\right\| \leqq t^{*}-t_{n}, \quad n=0,1, \cdots
$$

Proof. Since $Q(s, \bar{s}, t, \bar{t}) \geqq 0$ and $B(s, t) \geqq 0$ for $s, \bar{s}, t, \bar{t} \geqq 0$, it follows from (3.6) that $0=t_{0} \leqq t_{1} \leqq t_{2} \leqq \cdots \leqq t^{*}$. We have 


$$
\left\|x_{1}-x_{0}\right\| \leqq t_{1} \leqq t^{*}
$$

by (3.7) and (3.3). We show by induction using the contractor inequality (3.1) with $x=x_{n-1}, \bar{x}=\bar{x}_{n-1}$ and $t=-F x_{n-1}$ that

$$
\begin{aligned}
& \text { (3.9) } \begin{aligned}
\left|F x_{n}\right| \leqq Q\left(\left\|x_{n}-x_{n-1}\right\|,\left\|x_{n}-\bar{x}_{n-1}\right\|,\left\|x_{n}-x_{0}\right\|,\left\|\bar{x}_{n-1}-x_{0}\right\|\right) \\
\leqq
\end{aligned} \\
& \leqq \\
& \leqq
\end{aligned}
$$

in virtue of (3.3), (3.2) and (3.6). It follows from (3.10) that $x_{n} \varepsilon S$. We conclude from (3.11) that

$$
\left\|x_{n+p}-x_{n}\right\| \leqq t_{n+p}-t_{n} \text { for } n, p=1,2, \cdots
$$

Hence, the sequence $\left\{x_{n}\right\}$ converges to some element $x$ and we have $F x_{n} \rightarrow 0$ as $n \rightarrow \infty$, by (3.9). Since $F$ is closed, $F x=0$. The error estimate (3.8) results from (3.12) by letting $p \rightarrow \infty$ in (3.12).

Our further discussion of general iterative procedures (3.3) is based on certain lemmas concerning Newton-type iterative methods in one dimensional space. These methods are used in order to establish the convergence of the sequences $\left\{t_{n}\right\}$ in (3.6) (see Altman (1974)).

LEMMA 3.1. Altman (1974). Let $u(t)$ be a real valued function continuous on $\left[0, t^{*}\right]$ where $t^{*}$ is the smallest positive root of $u(t)=0$. Let $v(t)>0$ for $0 \leqq t<t^{*}$ be continuous on $\left[0, t^{*}\right]$. Suppose that

$$
u(s)-u(t)+v(t)(s-t)>0
$$

for arbitrary $0 \leqq t<s \leqq t^{*}$. Then all $t_{n}<t^{*}$ and the sequence $\left\{t_{n}\right\}$ defined by

$$
t_{n+1}=t_{n}+u\left(t_{n}\right) / v\left(t_{n}\right), \quad n=0,1, \cdots
$$

with initial values $t_{0}=0, t_{1}=\eta<t^{*}$ converges toward $t^{*}$ and satisfies the difference equations 


$$
t_{n+1}-t_{n}=\left[1 / v\left(t_{n}\right)\right]\left[u\left(t_{n}\right)-u\left(t_{n-1}\right)+v\left(t_{n-1}\right)\left(t_{n}-t_{n-1}\right)\right] .
$$

LEMMA 3.2. Altman (1974). Let $u$ be a real valued function with continuous second derivative $u^{\prime \prime}(t)>0$ for $0 \leqq t \leqq t^{*}$, where $t^{*}$ is the smallest positive root of $u(t)=0$. Let $v(t)>0$ for $0 \leqq t<t^{*}$ be continuous on $\left[0, t^{*}\right]$. If $\left|u^{\prime}(t)\right| \leqq v(t)$ for $0 \leqq t \leqq t^{*}$, then all $t_{n}<t^{*}$ and the sequence $\left\{t_{n}\right\}$ defined by (3.14) converges to $t^{*}$ and satisfies (3.15).

LEMMA 3.3. Altman (1974). Assume that $p_{i} \geqq 0, i=1, \cdots, 4, p_{1}>0$ and $p_{2}<1$. Let $0<\eta \leqq\left(1-p_{2}\right)^{2} / 4 \bar{p}_{1}$ where $\bar{p}_{1}=\max \left[p_{1},\left(p_{3}+p_{4}\right) / 2\right]$. Then the sequence $\left\{t_{n}\right\}$ defined by (3.14) with $u(t)=\bar{p}_{1} t^{2}-\left(1-p_{2}\right) t+\eta$ and $v(t)=1-p_{4} t$ is strictly increasing and is the same as that defined by

$$
t_{n+1}=t_{n}+\left[1 /\left(1-p_{4} t_{n}\right)\right]\left[\bar{p}_{1}\left(t_{n}-t_{n-1}\right)+p_{2}+\left(2 \bar{p}_{1}-p_{4}\right) t_{n-1}\right]\left(t_{n}-t_{n-1}\right)
$$

with initial values $t_{0}=0, t_{1}=\eta$ and we have

$$
\lim _{n \rightarrow \infty} t_{n}=t^{*}=\left\{\left(1-p_{2}\right)-\left[\left(1-p_{2}\right)^{2}-4 p_{1} \eta\right]^{\frac{1}{3}}\right\} / 2 \bar{p}_{1} .
$$

The sequence $\left\{t_{n}\right\}$ majorizes the sequence $\left\{s_{n}\right\}$, i.e.,

$$
\begin{gathered}
s_{n+1}-s_{n} \leqq t_{n+1}-t_{n}, \quad n=0,1, \cdots, \text { where } \\
s_{n+1}=s_{n}+\left[1 /\left(1-p_{4} s_{n}\right)\right]\left[p_{1}\left(s_{n}-s_{n-1}\right)+p_{2}+p_{3} s_{n-1}\right]\left(s_{n}-s_{n-1}\right), n=0,1, \cdots
\end{gathered}
$$

Lemma 3.4. (Ortega and Rheinboldt (1970), 12.6.2) Let $a, \eta$ be positive constants such that $h=a \eta \leqq \frac{1}{2}$. Then the sequence $\left\{t_{n}\right\}$ defined by

$$
t_{n+1}=t_{n}+a\left(t_{n}-t_{n-1}\right)^{2} / 2\left(1-a t_{n}\right), \quad n=0,1, \cdots,
$$

$t_{0}=0, t_{1}=\eta$, converges to the smaller root $t^{*}$ of equation

$$
\frac{1}{2} a t^{2}-t+\eta=0
$$

and the error estimate holds

$$
t^{*}-t_{n} \leqq\left(a 2^{n}\right)^{-1}(2 h)^{2^{n}}, \quad n=0,1, \cdots .
$$

The sequence defined by (3.18) is the same as that defined by

$$
t_{n+1}=t_{n}+\left(\frac{1}{2} a t_{n}^{2}-t_{n}+\eta\right) /\left(1-a t_{n}\right)
$$

or by

$$
t_{n+1}=\left(\frac{1}{2} a t_{n}^{2}-\eta_{n} /\left(a t_{n}-1\right), \quad t_{0}=0 .\right.
$$

THEOREM 3.2. Let $F: D \rightarrow R$ be a closed non-linear functional with domain $D$ containing $S$ and let $\Gamma: D \times D \rightarrow X^{*}$ be a two-point contractor satisfying (3.1) and (3.2). Assume that there exist two functions $u(t)$ and $v(t)$ satisfying the hypotheses of Lemma (3.1) or (3.2) and such that 


$$
\begin{aligned}
& B\left(s, \gamma_{2} s\right) Q\left(s-t, \gamma_{1}(s-t), t, \gamma_{2} t\right) \\
& \quad \leqq[1 / v(s)][u(s)-u(t)+v(t)(s-t)]
\end{aligned}
$$

for $0 \leqq t<s \leqq t^{*}$, where $t^{*}$ is the smallest positive root of $u(t)=0$ and

$$
Q\left(0,0, t^{*}, \gamma_{2} t^{*}\right)=0 .
$$

Finally let $x_{0} \varepsilon D$ be such that it satisfies (3.7). Then all $x_{n}$ lie in $S$ and the sequence $\left\{x_{n}\right\}$ defined by (3.3)-(3.5) converges to a solution $x$ of $F x=0$. The error estimate (3.8) holds, where the sequence $\left\{t_{n}\right\}$ is defined by (3.14) or (3.15) provided that $\left\|F x_{0} \Gamma\left(x_{0}, \bar{x}_{0}\right)\right\| \leqq \eta$.

Proof. Using the contractor inequality (3.1) with $x=x_{n-1}, \bar{x}=\bar{x}_{n-1}$ and $t=-F x_{n-1}$ we prove by induction the inequalities (3.9)-(3.12) exactly in the same way as in the proof of Theorem 3.1. In virtue of (3.22) we obtain

$$
\begin{aligned}
& \left\|x_{n+1}-x_{n}\right\|=\| F x_{n} \Gamma\left(x_{n}, \bar{x}_{n}\|\leqq\| \Gamma\left(x_{n}, \bar{x}_{n}\right) \| \cdot\left|F x_{n}\right|\right. \\
& \leqq B\left(\left\|x_{n}-x_{0}\right\|,\left\|\bar{x}_{n}-\bar{x}_{0}\right\|\right) Q\left(\left\|x_{n}-x_{n-1}\right\|,\left\|x_{n}-\bar{x}_{n-1}\right\|,\right. \\
& \left.\left\|x_{n}-x_{0}\right\|,\left\|\bar{x}_{n}-\bar{x}_{0}\right\|\right) \\
& \leqq B\left(\left\|x_{n}-x_{0}\right\|, \gamma_{2}\left\|x_{n}-x_{0}\right\|\right) Q\left(\left\|x_{n}-x_{n-1}\right\|, \gamma_{2}\left\|x_{n}-x_{n-1}\right\|\right. \text {, } \\
& \left.\left\|x_{n}-x_{0}\right\|, \gamma_{2}\left\|\bar{x}_{n}-\bar{x}_{0}\right\|\right) \\
& \leqq B\left(t_{n}, \gamma_{2} t_{n}\right) Q\left(t_{n}-t_{n-1}, \gamma_{1}\left(t_{n}-t_{n-1}\right), t_{n-1}, \gamma_{2} t_{n-1}\right) \\
& \leqq\left[1 / v\left(t_{n}\right)\right]\left[u\left(t_{n}\right)-u\left(t_{n-1}\right)+v\left(t_{n-1}\right)\left(t_{n}-t_{n-1}\right)\right]=t_{n+1}-t_{n} \text {. }
\end{aligned}
$$

Hence, we have

and

$$
\begin{gathered}
\left\|x_{n+1}-x_{n}\right\| \leqq t_{n+1}-t_{n} \\
\left\|x_{n+1}-x_{0}\right\| \leqq t_{n+1}<t^{*}=r, \quad n=0,1, \cdots
\end{gathered}
$$

By Lemma 3.1 or 3.2, the sequence $\left\{t_{n}\right\}$ defined by (3.14) or (3.15) is increasing and converges toward the smallest positive root $t^{*}$ of $u(t)=0$. In virtue of (3.24), the sequence $\left\{x_{n}\right\}$ defined by (3.3)-(3.5) converges to some element $x$. It follows from (3.25) that $x_{n}$ is in $S$ and $F x_{n} \rightarrow 0$ as $n \rightarrow \infty$ in virtue of (3.9) and (3.23). Since $F$ is closed, we conclude that $F x=0$. The error estimate (3.8) follows from (3.24).

As a special case of Theorem 3.2 we obtain the following

COROLLARY 3.1. In addition to the hypotheses of Theorem 3.2 assume instead of $(3,22)$ that

$$
\begin{aligned}
& B\left(s, \gamma_{2} s\right) Q\left(s-t, \gamma_{1}(s-t), t, \gamma_{2} t\right) \\
& \quad \leqq\left[1 /\left(1-p_{4} s\right)\right]\left[p_{1}(s-t)+p_{2}+p_{3} t\right](s-t)
\end{aligned}
$$


for $0 \leqq t<s \leqq t^{*}$ and $u(t)=\bar{p}_{1} t^{2}-\left(1-p_{2}\right) t+\eta$ and $v(t)=1-p_{4} t$ satisfy the conditions of Lemma 3.3. Then all assertions of Theorem 3.2 hold provided that the sequence $\left\{t_{n}\right\}$ and $t^{*}$ are defined as in Lemma 3.3.

Proof. The proof is exactly the same as that of Theorem 3.2.

As a special case of Corollary 3.1 we obtain the following

COROLLARY 3.2. Under the hypotheses of Corollary 3.1 instead of (3.22) assume that

$$
Q\left(s-t, \gamma_{1}(s-t), t, \gamma_{2} t\right) \leqq\left[\frac{1}{2} K(s-t)+d_{0}+d_{1} t\right](s-t)
$$

with $d_{0}, d_{1} \geqq 0$ and

$$
B\left(s, \gamma_{2} s\right) \leqq B /(1-\alpha s)
$$

in (3.2) with $B d_{0}<1$ and $\eta \leqq\left(1-B d_{0}\right)^{2} / 4 p_{1}$, where $\bar{p}_{1}=\max \left[p_{1},\left(p_{3}+p_{4}\right) / 2\right]$.

Then all assertions of Theorem 3.2 hold provided that the sequence $\left\{t_{n}\right\}$ and $t^{*}$ are defined as in Lemma 3.3 with $p_{1}=\frac{1}{2} B K, p_{2}=B d_{0}, p_{3}=B d_{1}$ and $p_{4}=x$.

Proof. Since condition (3.26) is fulfilled, the hypotheses of Corollary 3.1 are satisfied.

\section{Approximate first ordered divided differences}

Let $F: D \rightarrow R$ be a non-linear functional with domain $D$ containing the sphere $S=S\left(x_{0}, r\right)$. Assume that the first divided difference functional $G(x, \bar{x})$ of $F$ exists for $x, \bar{x} \varepsilon S$ and satisfies

$$
|F x-F \bar{x}-G(\xi, \bar{x})(x-\bar{x})| \leqq K\|x-\bar{x}\| \cdot\|x-\xi\|
$$

for $x, \bar{x} \varepsilon S$. Let $J: D \times D \rightarrow X^{*}$ be a mapping such that

$$
\|G(x, \bar{x})-J(x, \bar{x})\| \leqq d_{0}+\bar{d}_{1}\left[\left\|x-x_{0}\right\|+\left\|\bar{x}-\bar{x}_{0}\right\|\right]
$$

for $x, \bar{x} \varepsilon S$.

Lemma 4.1. For each $x, \bar{x} \varepsilon S$ let $y \varepsilon X$ be such that $J(x, \bar{x}) y \neq 0$ and put $\Gamma(x, \bar{x})=y / J(x, \bar{x}) y$. Then $\Gamma: S \times S \rightarrow X$ is a two-point contractor for $F$ satisfying the following contractor inequality

$$
\begin{aligned}
& |F(x+t \Gamma(x, \bar{x}))-F x-t| \\
& \quad \leqq R\|t \Gamma(x, \bar{x})\|\left\{\|x+t \Gamma(x, \bar{x})-\bar{x}\|+d_{0}+d_{1}\left(\left\|x-x_{0}\right\|+\left\|\bar{x}-\bar{x}_{0}\right\|\right)\right\}
\end{aligned}
$$

for $x, \bar{x} \varepsilon S$ whenever $x+t \Gamma(x, \bar{x}) \varepsilon S$.

Proof. We have in virtue of (4.1) and (4.2) 


$$
\begin{aligned}
& |F(x+t \Gamma(x, \bar{x}))-F x-t| \leqq|F(x+t \Gamma(x, \bar{x}))-F x-G(\bar{x}, x) t \Gamma(x, \bar{x})| \\
& \quad+|[G(x, \bar{x})-J(x, \bar{x})] t \Gamma(x, \bar{x})| \leqq R\|t \Gamma(x, \bar{x})\| \cdot\|x+t \Gamma(x, \bar{x})-\bar{x}\| \\
& \quad+\left\{d_{0}+d_{1}\left(\left\|x-x_{0}\right\|+\left\|x-x_{0}\right\|\right)\right\}\|t \Gamma(x, \bar{x})\| .
\end{aligned}
$$

Now let us discuss the iterative procedure (3.3) under condition (1.7) to be shown as a special case of condition (3.4).

LEMMA 4.2. Suppose that there exists a constant number $\delta$ such that

$$
\|J(x, \bar{x})\| \leqq \delta \text { for } x, \bar{x} \varepsilon S .
$$

Then condition (1.7) implies (3.4) with $\gamma_{1}=1+\delta C$.

Proor. It follows from (4.4) that

$$
1 /\|\Gamma(x, \bar{x})\|=|J(x, \bar{x}) y| /\|y\| \leqq \delta \text { for } x, \bar{x} \varepsilon S .
$$

Hence, we obtain in virtue of (1.7) and (4.5)

$$
\begin{aligned}
& \left\|x_{n}-\bar{x}_{n}\right\| \leqq C\left|F x_{n}\right|=C\left\|F x_{n} \Gamma\left(x_{n}, \bar{x}_{n}\right)\right\| / \| \Gamma\left(x_{n}, \bar{x}_{n} \|\right. \\
& \leqq \delta C\left\|x_{n+1}-x_{n}\right\| \text { for } n=0,1, \cdots \text { provided that } x_{n} \varepsilon S \text {. Since } \\
& \left\|x_{n+1}-\bar{x}_{n}\right\|=\left\|x_{n}-F x_{n} \Gamma\left(x_{n}, \bar{x}_{n}\right)-\bar{x}_{n}\right\| \\
& \leqq\left\|x_{n}-\bar{x}_{n}\right\|+\left\|F x_{n} \Gamma\left(x_{n}, \bar{x}_{n}\right)\right\| \leqq(1+\delta C)\left\|x_{n+1}-x_{n}\right\|,
\end{aligned}
$$

in virtue of (4.6), we conclude that condition (3.4) with $\gamma_{1}=1+\delta C$ results from (1.7) and (4.4).

Suppose now that there exists a constant $\bar{M}$ such that

$$
\left\|J(x, \bar{x})-J\left(x_{0}, \bar{x}_{0}\right)\right\| \leqq \bar{M}\left[\left\|x-x_{0}\right\|+\left\|\bar{x}-\bar{x}_{0}\right\|\right] \text { for } x, \bar{x} \varepsilon S .
$$

Let $\beta<1$ be any positive fixed number and for each pair $x, \bar{x} \varepsilon S$ choose $y \varepsilon X$ so as to satisfy

$$
J(x, \bar{x}) y \geqq \beta\|J(x, \bar{x})\| \text { and }\|y\|=1 .
$$

Then we obtain

$$
\begin{aligned}
& \|J(x, \bar{x})\| \geqq\left\|J\left(x_{0}, \bar{x}_{0}\right)\right\|\left|1-\left[\left\|J\left(x_{0}, \bar{x}_{0}\right)-J(x, \bar{x})\right\|\right] /\left\|J\left(x_{0}, \bar{x}_{0}\right)\right\|\right| \\
& \geqq\left\|J\left(x_{0}, \bar{x}_{0}\right)\right\|\left(1-\bar{B} \bar{M}\left[\left\|x-x_{0}\right\|+\left\|\bar{x}-\bar{x}_{0}\right\|\right]\right) \geqq\left(1-B M\left\|x-x_{0}\right\|\right) / B
\end{aligned}
$$

provided that $\left\|\bar{x}-\bar{x}_{0}\right\| \leqq \gamma_{2}\left\|x-x_{0}\right\|$,

$$
1 /\left\|J\left(x_{0}, \bar{x}_{0}\right)\right\| \leqq \bar{B},
$$

and $B=\bar{B} / \beta, M=\bar{M}\left(1+\gamma_{2}\right), B M r<1$.

Then we obtain in virtue of (4.8) and (4.9) 


$$
\|\Gamma(x, \bar{x})\| \leqq B /\left(1-B M\left\|x-x_{0}\right\|\right) \text { for } x, \bar{x} \varepsilon S \text { if } B M r \leqq 1 .
$$

TheOREM 4.1. Let $F: D \rightarrow R$ be a closed non-linear functional with domain $D$ containing $S$. Assume that the first divided difference functional of $F$ exists and satisfies (4.1), (4.2) and (4.4). Let $x_{0}, \bar{x}_{0}$ be chosen so as to satisfy (4.9) and

Let

$$
\left\|F x_{0} \Gamma\left(x_{0}, \bar{x}_{0}\right)\right\| \leqq \eta \text { and } B d_{0}<1 .
$$

$$
\eta \leqq\left(1-B d_{0}\right)^{2} / 4 \bar{p}_{1}, K=2 R(1+\delta C), M=\bar{M}\left(1+\gamma_{2}\right), p_{4}=B M
$$

and set $r=t^{*}$ where $t^{*}$ is defined as in Lemma 3.3 with

$$
p_{1}=\frac{1}{2} B K, p_{2}=B d_{0}, p_{3}=B d_{1}, d_{1}=d_{1}\left(1+\gamma_{2}\right) \text {. }
$$

Then the sequence $\left\{x_{n}\right\}$ defined by (3.3) under condition (1.7) remains in $S$ and converges to a solution $x$ of $F x=0$.

Proof. It follows from Lemma 4.1 that $\Gamma$ is a two-point contractor for $F$ satisfying the contractor inequality (4.3). Since $M t^{*} \leqq 1 / B, \Gamma$ satisfies (4.10). It results from (4.3) and (4.6) that

$$
\begin{aligned}
& |F(x+t \Gamma(x, \bar{x}))-F x-t| \leqq R(1+\delta C)\|t \Gamma(x, \bar{x})\|^{2} \\
& \quad+\left\{d_{0}+\bar{d}_{1}\left(1+\gamma_{2}\right)\left\|x-x_{0}\right\|\right\}\|t \Gamma(x, \bar{x})\|
\end{aligned}
$$

for $t=-F x,\|x-\bar{x}\| \leqq C \mid F x\|,\| \bar{x}-\bar{x}_{0}\left\|\leqq \gamma_{2}\right\| x-x_{0} \|$.

Hence, we obtain that condition (3.27) is satisfied with $\gamma_{1}=1+\delta C, K=2 R \gamma_{1}$ and $d_{1}=d_{1}\left(1+\gamma_{2}\right)$. Thus, the hypotheses of Corollary 3.2 are satisfied and the proof is complete.

\section{Quadratic convergence}

A special case of 'Theorem 3.1 will be discussed. This case yields quadratic convergence and is based on Lemma 3.4.

THEOREM 5.1. Let $F: D \rightarrow R$ be a closed non-linear functional with domain $D$ containing the sphere $S=S\left(x_{0}, r\right)$. Let $\Gamma: D \times D \rightarrow X^{*}$ be a two-point contractor for $F$ satisfying the contractor inequality

$$
|F(x+t \Gamma(x, \bar{x}))-F x-t| \leqq K\|t \Gamma(x, \bar{x})\| \cdot\|x+t \Gamma(x, \bar{x})-\bar{x}\|
$$

and

$$
\|\Gamma(x, \bar{x})\| \leqq B /\left[1-M\left(\left\|x-x_{0}\right\|+\left\|\bar{x}-\bar{x}_{0}\right\|\right)\right]
$$

for $x, \bar{x} \varepsilon S$ whenever $x+t \Gamma(x, \bar{x}) \varepsilon S$.

Put $a=\max \left[2 B \gamma_{1} K, M\left(1+\gamma_{2}\right)\right]$ and assume that

$$
h=a \eta \leqq \frac{1}{2} \text { and } r=t^{*}=\left[1-\left(1-2 h^{\frac{1}{2}}\right] / a\right. \text {. }
$$


Let $x_{0}, \bar{x}_{0} \varepsilon D$ be chosen so as to satisfy

$$
\left\|F x_{0} \Gamma\left(x_{0}, \bar{x}_{0}\right)\right\| \leqq \eta .
$$

Then all $x_{n}$ lie in $S$ and the sequence $\left\{x_{n}\right\}$ defined by (3.3)-(3.5) converges to a solution $x$ of $F x=0$ and the error estimate holds.

$$
\left\|x-x_{n}\right\| \leqq t^{*}-t_{n} \leqq\left(a 2^{n}\right)^{-1}(2 h)^{2 n},
$$

for $n=0,1, \cdots$, where the sequence $\left\{t_{n}\right\}$ is defined as in Lemma 3.4.

Proof. We show by induction that

$$
\left|F x_{n}\right| \leqq K \gamma_{1}\left\|F x_{n-1} \Gamma\left(x_{n-1}, \bar{x}_{n-1}\right)\right\|^{2}=K \gamma_{1}\left\|x_{n}-x_{n-1}\right\|^{2}
$$

in virtue of (3.3), (3.4) and (5.1) with $x=x_{n-1}, \bar{x}=\bar{x}_{n-1}$ and $t=-F x_{n-1}$. It follows from (5.2) and (3.5) that

$$
\left\|\Gamma\left(x_{n}, \bar{x}\right)\right\| \leqq B /\left(1-M\left(1+y_{2}\right)\left\|x-x_{0}\right\|\right) .
$$

Hence, we obtain by induction

$$
\begin{aligned}
& \left\|x_{n+1}-x_{n}\right\| \leqq B K \gamma_{1}\left\|x_{n}-x_{n-1}\right\|^{2} /\left(1-M\left(1+\gamma_{2}\right)\left\|x_{n}-x_{0}\right\|\right) \\
& \quad \leqq \frac{1}{2} a\left\|x_{n}-x_{n-1}\right\|^{2} /\left(1-a\left\|x_{n}-x_{0}\right\|\right) \leqq \frac{1}{2} a\left(t_{n}-t_{n-1}\right)^{2} /\left(1-a t_{n}\right)=t_{n+1}-t_{n}
\end{aligned}
$$

and

$$
\left\|x_{n}-x_{0}\right\| \leqq \sum_{i=1}^{n}\left\|x_{i}-x_{i-1}\right\| \leqq \sum_{i=1}^{n}\left(t_{n}-t_{n-1}\right)=t_{n} \leqq t^{*},
$$

where the sequence $\left\{t_{n}\right\}$ is the same as that defined in Lemma 3.4. Now using this lemma we continue the same argument as in the proof of Theorem 3.1.

Let us discuss a special case of Theorem 5.1 where the two-point contractor $\Gamma$ is generated by the first divided difference functional $G$ of $F$. Thus, we consider the iterative procedure

$$
x_{n+1}=x_{n}-F x_{n} \Gamma\left(x_{n}, \bar{x}_{n}\right), \quad n=0,1, \cdots,
$$

where $\Gamma\left(x_{n}, \bar{x}_{n}\right)=y_{n} / G\left(x_{n}, \bar{x}_{n}\right) y_{n}$ with $y_{n}$ chosen so as to satisfy

$$
G\left(x_{n}, \bar{x}_{n}\right) y_{n} \geqq \beta \| G\left(x_{n}, \bar{x}_{n}\right) \text { and }\left\|y_{n}\right\|=1 \text {, }
$$

where $\beta<1$ is any positive fixed number. We assume also that $G$ satisfies the following condition

$$
\|G(x, \bar{x})-G(\xi, \bar{\xi})\| \leqq K[\|x-\xi\|+\|\bar{x}-\bar{\xi}\|]
$$

for $x, \bar{x}, \xi, \bar{\xi} \varepsilon D$. Suppose that

$$
1 /\left\|G\left(x_{0}, \bar{x}_{0}\right)\right\| \leqq \tilde{B}
$$


and

$$
B=\bar{B} / \beta, \bar{M}=K\left(1+\gamma_{2}\right), B M r \leqq 1
$$

Then we obtain

$$
\begin{aligned}
& \|G(x, \bar{x})\| \geqq\left\|G\left(x_{0}, \bar{x}_{0}\right)\right\|\left|1-\left[\left\|G\left(x_{0}, \bar{x}_{0}\right)-G(x, \bar{x})\right\|\right] /\left\|G\left(x_{0}, \bar{x}_{0}\right)\right\|\right| \\
& \geqq\left\|G\left(x_{0}, \bar{x}_{0}\right)\right\|\left(1-\bar{B} K\left[\left\|x-x_{0}\right\|+\left\|\bar{x}-\bar{x}_{0}\right\|\right]\right) \geqq\left(1-B \bar{M}\left\|x-x_{0}\right\|\right) / B
\end{aligned}
$$

in virtue of (5.8) and (5.9). Hence, we conclude from (5.7) that

$$
\|\Gamma(x, \bar{x})\| \leqq B /\left(1-B \bar{M}\left\|x-x_{0}\right\|\right) \text { for } x, \bar{x} \varepsilon S \text { if } B M r \leqq 1 .
$$

THEOREM 5.2. Let $F: D \rightarrow R$ be a non-linear functional with domain $D$ containing the sphere $S=S\left(x_{0}, r\right)$. Suppose that the first divided difference functional $G$ of $F$ exists and satisfies conditions (5.8) and (5.9). Put $a=B K \max \left[2 \gamma_{1}, 1+\gamma_{2}\right]$ and assume that conditions (5.3) and (5.4) are satisfied. Then all $x_{n}$ lie in $S$ and the sequence $\left\{x_{n}\right\}$ defined by (5.6), (3.4) and (3.5) converges to a root $x$ of $F x=0$ and the error estimate (5.5) holds.

ProOF. It follows from (5.8) that $\Gamma$ is a two-point contractor for $F$ satisfying the contractor inequality (5.1). We infer from (5.10) that (5.2) is also satisfied with $M$ replaced by $B K$. Hence, it follows that all hypotheses of Theorem 5.1 are satisfied and the proof follows immediately.

Consider now the iterative procedure defined by (5.6) and (1.7). We obtain the following theorem as a special case of Theorem 5.2.

THEOREM 5.3. Under the hypotheses of Theorem 5.2 let the sequence $\left\{x_{n}\right\}$ be defined by (5.6), (1.7) and (3.5). If, in addition,

$$
\|G(x, \bar{x})\| \leqq \delta \quad \text { for } \quad x, \bar{x} \varepsilon S,
$$

then all assertions of Theorem 5.2 hold with $\gamma_{1}=1+\delta C$.

Proof. It remains to prove that condition (3.4) is satisfied. This follows from Lemma 4.1 with $J(x, \bar{x})$ replaced by $G(x, \bar{x})$. Then we conclude that condition (3.4) is satisfied with $\gamma_{1}=1+\delta C$.

\section{References}

M. Altman (1973), 'Inverse differentiability, contractors and equations in Banach spaces', Studia Math., Vol. 46.

M. Altman (1974), 'Contractors with non-linear majorant functions and equations in Banach spaces', Boll. U. M. I., 4 (9). 
M. Altman, 'Contractors and secant methods for solving equations', (to appear).

M. Altman, 'Contractors and roots of non-linear functionals', (to appear (a)).

J. M. Ortega and W. C. Rheinboldt (1970), Iterative Solutions of Non-linear Equations in Several Variables, (Acad. Press, 1970.)

Department of Mathematics

University of Newcastle

New South Wales, 2308

Australia.

Louisiana State University

Baton Rouge

Louisiana 70803

U. S. A. 